\title{
Una revisión al uniformitarismo, sus aplicaciones en paleontología y las parti- cularidades del registro fósil de animales terrestres mexicanos
}

\author{
A review of uniformitarianism, its applications in paleontology and the par- \\ ticularities of the fossil record of Mexican land animals
}

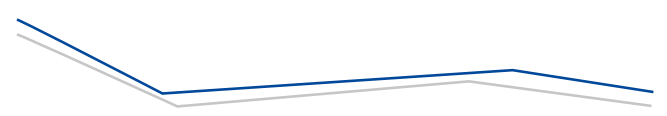

Rosalía Guerrero-Arenas*凶, Eduardo Jiménez-Hidalgo*, Víctor Manuel Bravo-Cuevas**

Guerrero-Arenas, R., Jiménez-Hidalgo, E., \& Bravo-Cuevas, V. M. (2020). Una revisión al uniformitarismo, sus aplicaciones en paleontología y las particularidades del registro fósil de animales terrestres mexicanos. Investigación y Ciencia de la Universidad Autónoma de Aguascalientes, 28(81), 74-81.

El estudio del registro fósil permite formular hipótesis sobre los ambientes del pasado. Las comparaciones con análogos actuales se basan en el estudio de diversos indicadores. El objetivo del artículo es discutir la validez de dichas hipótesis sobre las condiciones ambientales del pasado. También se argumenta por qué el uniformitarismo, como concepto, puede ser malentendido y mal aplicado en varios estudios, originando interpretaciones débiles. Se discuten las razones de la falta de interpretaciones válidas de los ambientes del pasado del territorio mexicano, principalmente la carencia de análogos modernos. A partir de ejemplos con organismos terrestres fósiles se argumenta la singularidad del registro mexicano y las limitaciones existentes para la interpretación de sus ambientes del pasado y su biocronología.

Palabras clave: fósiles; actualismo; paleoambientes; paleobiología; mamíferos fósiles; neontología.

Keywords: fossils; actualism; paleoenvironments; paleobiology; fossil mammals; neontology.

Recibido: 11 de diciembre de 2019, aceptado: 22 de septiembre de 2020

* Laboratorio de Paleobiología, Universidad del Mar campus Puerto Escondido, Oaxaca, México. Ciudad Universitaria, Carretera Vía Sola de Vega, Puerto Escondido, San Pedro Mixtepec, C. P. 71980, Juquila, Oax. México. Correo electrónico: rosaliaga@zicatela.umar.mx; eduardojh@ zicatela.umar.mx ORCID: http://orcid.org/0000-0002-5763-806X; http:// orcid.org/0000-0002-6372-0625

** Museo de Paleontología, Área Académica de Biología, Ciudad del Conocimiento, Universidad Autónoma del Estado de Hidalgo. Kilómetro 4.5 carretera Pachuca-Tulancingo, Colonia Carboneras, Mineral de la Reforma, C. P. 42184, Hidalgo, México. Correo electrónico: vbravo@ vaeh.edu.mx ORCID: http://orcid.org/0000-0002-6395-7147
The study of fossil record allows the proposal of hypotheses about past environments. Comparisons with recent analogues are based on the study of several proxies. The aim of the article is to discuss examples the reliability of those hypotheses about past environmental conditions. Also, is discussed why the concept of uniformitarianism could be misunderstood and wrongly applied in several studies, leading weak interpretations. Several causes of lack of valid interpretations of ancient environments in Mexican territory are discussed, mainly the absence of modern analogues. Based on fossil terrestrial organisms examples, the singularity of the Mexican fossil record is discussed, as well as the limitations in the interpretation of past environments and biochronology.

\section{INTRODUCCIÓN}

La información derivada del estudio del registro fósil ha demostrado ser una fuente confiable para inferir las condiciones ambientales del pasado, así como la historia evolutiva de taxones desaparecidos. La formulación de las hipótesis se rige por el uniformitarismo, principio que en las geociencias indica que los procesos actuales operaron en el pasado y que son clave para interpretar eventos pasados (Cronin, 2010). Sin embargo, a veces las interpretaciones no son objetivas.

Con el fin de que futuros profesionales de las ciencias de la Tierra se familiaricen con el uso del uniformitarismo en interpretaciones paleobiológicas, este texto aborda cuatro objetivos: a) revisar el uso de 
este concepto en las ciencias de la Tierra, b) explicar por qué el registro fósil mexicano puede ser particular y sin análogos, c) mencionar algunos ejemplos de la paleobiota mexicana terrestre y su falta de analogía con biotas sincrónicas en América del Norte, y d) demostrar que el avance en el conocimiento de los paleoambientes depende del avance conjunto de la neontología y la paleontología.

\section{El uniformitarismo: Un concepto mal entendido}

El análisis de la evidencia fósil parte de que el conocimiento de factores que influyen en la abundancia y distribución de los organismos modernos permite realizar inferencias sobre los controles ambientales dominantes en las poblaciones del pasado. Si se aplica este enfoque a los conjuntos de fósiles, si se contara con equivalentes actuales permitiría reconstruir las condiciones ambientales del pasado con un grado razonable de confianza (Lowe \& Walker, 2015). La frase "El presente es la llave del pasado" se repite incansablemente en diversos libros y en artículos indizados. Sin embargo, las interpretaciones de cómo vivían las biotas pasadas -a partir de comparaciones con actuales- no son tan simples.

Uno de los errores más comunes es la comprensión del concepto del uniformitarismo. El término fue acuñado en 1832 por William Whewell (Romano, 2015; Shea, 1982) y ha sido modificado consecuentemente. Desde el punto de vista filosófico varios autores lo consideran problemático, ya que la acepción original ha variado a lo largo del tiempo (Baker, 2014; García-Cruz, 1999; Gould, 1965; Gould, 1987; Knight \& Harrison, 2014; Romano, 2015; Shea, 1982). De acuerdo con Gould (1965) y Romano (2015) el uniformitarismo sustantivo (substantive uniformitarianism, en su acepción original) considera que los procesos naturales operan y siempre han operado en tasas uniformes, produciendo cambios lentos y acumulables. Por otro lado, el uniformitarismo metodológico (methodological uniformitarianism) considera a priori que las leyes naturales son invariantes en tiempo y en espacio.

En cuanto a la uniformidad de los procesos del pasado y su permanencia en el presente, Balashov (1994) identificó al uniformitarismo débil y al fuerte. En el uniformitarismo débil (weak uniformitarianism) se requiere que los tipos de procesos que funcionan en el pasado geológico sean los mismos que en el presente, lo cual implica una temporalidad uniforme en las leyes naturales; el uniformitarismo fuerte (strong uniformitarianism) va más allá y demanda que no solamente los tipos de procesos, sino las intensidades sean las mismas en el pasado y en el presente (Balashov, 1994).

Como resultado de estas revisiones se recomienda que el enfoque del actualismo se use con cautela (Harrison, 2012). Aunque el registro fósil es la evidencia más fehaciente de la historia de la vida de nuestro planeta, también representa una versión imperfecta de dicho pasado (Louys, Wilkinson, \& Bishop, 2012), ya que no todo lo que alguna vez vivió se encuentra preservado en el registro geológico. Debido a que la Tierra es un sistema en constante cambio, el principio del uniformitarismo no puede emplearse indistintamente.

Knight \& Harrison (2014) discuten ampliamente que es imposible aplicar este principio en el presente y futuro, debido a que el Antropoceno -intervalo de tiempo en la historia de la Tierra en que vivimos actualmente, en el que muchos procesos elementales de la superficie terrestre son dominados por la influencia humana (Lluch-Cota, Saldívar-Lucio, \& Aranceta-Garza, 2017)- carece de análogos en el pasado geológico. La actividad humana actualmente dirige la dinámica de varios sistemas contemporáneos de la Tierra y modifica su comportamiento más allá de los límites de su variabilidad natural, por ello el Antropoceno debe considerarse como una singularidad en la que hay incertidumbre (es decir, hay escasa predictibilidad) y no es posible presagiar cómo funcionarán los sistemas del planeta-como el clima-debido a las acciones antropogénicas.

En ocasiones no es posible usar el principio del uniformitarismo debido a la falta de analogía con grupos fósiles y recientes. Se ha enfatizado que aun cuando las comunidades de plantas fósiles mexicanas presentan una semejanza taxonómica con alguna comunidad actual, siempre hay diferencias (Cevallos-Ferriz, González-Torres, \& Calvillo-Canadell, 2012). Caballero-Rodríguez, Lozano-García y Correa-Metrio (2017) mencionan que la vegetación del centro de México durante el Pleistoceno carece de análogos con respecto a ecosistemas locales recientes. Con relación a la fauna, las asociaciones disarmónicas del Pleistoceno (conjuntos de especies con representantes de regiones templadas y tropicales) ejemplifican esta 
issn 1665-4412, e-issn 2521-9758

Guerrero-Arenas, R., Jiménez-Hidalgo, E., \& Bravo-Cuevas, V. M. falta de analogía, como las faunas pleistocénicas de la cueva de San Josecito, Nuevo León, y de Valsequillo, Puebla (Ceballos, Arroyo-Cabrales, \& Ponce, 2010).

Otra limitación de la aplicación del uniformitarismo depende de la información biológica sobre los diversos taxones, tanto fósiles como recientes. Por ejemplo, De Francesco (2013) observó que el uniformitarismo tiene limitaciones con moluscos dulceacuícolas debido a los problemas de identificación de sus especies, la escasa información ecológica disponible y los amplios rangos de tolerancia ecológica que tienen especies recientes.

\section{Las particularidades del registro fósil mexicano}

La biota fósil de México, al igual que la reciente, se caracteriza por una vasta biodiversidad. Las razones ecológicas y geológicas de dicha biodiversidad han sido revisadas ampliamente por varios autores (Cevallos-Ferriz \& González-Torres, 2005; FloresVillela \& Martínez-Salazar, 2009; Ramírez-Barahona \& Eguiarte, 2013). La historia geológica de México es distinta a la del resto de América del Norte; Ferrari (2017) señala que los procesos tectónicos regionales -orógenos y rupturas continentales-, el vulcanismo y los movimientos en masa que ha sufrido el planeta han influido en la construcción de barreras físicas y subdivisiones geomórficas continentales; así como nuevas vías de comunicación atmosférica, como en el clima, al producir cambios en la circulación atmosférica.

Las numerosas orogenias de nuestro territorio han creado diversos microhábitats, en los cuales las poblaciones se han desarrollado de manera distinta. Asumir que la evolución de las biotas de EE. UU. y México es muy semejante -y en algunos casos igualcomo se postuló desde finales del siglo pasado, originó varios supuestos erróneos de que algunas poblaciones y comunidades estaban dentro de un continuo temporal y espacial. En la siguiente sección se mostrará, con casos particulares del centro y sur de México, cómo las biotas mexicanas difieren del resto de las de América del Norte.

\section{No todo en América del Norte es "norteamericano"}

Además de los estudios de índole paleobiológica, los mamíferos también han sido utilizados para correlacionar estratos y circunscribir dichos estratos a la escala del tiempo geológico, mediante las
Edades de Mamíferos Terrestres (Lindsay, 2003; Woodburne, 2004). Éstas se definen como un intervalo relativamente corto de tiempo geológico que puede ser reconocido de otro intervalo temporal anterior o posterior, con base en un conjunto característico de taxa de mamíferos en una región específica (Lindsay, 2003). Las regiones geográficas de las edades de mamíferos más utilizados son muy amplias, por lo que existen distintos conjuntos de edades de mamíferos para América del Norte, América del Sur y Asia, por ejemplo (Hulbert, 2015).

En México, tradicionalmente se han utilizado las NALMA (North American Land Mammals Ages; Edades de Mamíferos Terrestres de Norteamérica) porque se considera que las faunas de México son equivalentes a las de EE. UU. Esto significa que las especies de los conjuntos de mamíferos mexicanos de una edad geológica determinada son las mismas que se pueden reconocer en el país vecino. Sin embargo, los estudios paleontológicos recientes donde se realiza un riguroso control estratigráfico de los ejemplares, dondesemuestreamacro-ymicrofauna-, asícomolos fechamientos radiométricos y magnetoestratigráficos de las capas fosiliferas comienzan a mostrar que esta generalización no es del todo acertada.

Los conjuntos mexicanos de mamíferos fósiles no siempre son equivalentes a los de EE. UU. ni las asociaciones identificadas son de la misma edad geológica. Por ejemplo, los antilocápridos Cosoryx y Plioceros se extinguen al final del Clarendoniano 19 $\mathrm{Ma}$ ) en las faunas locales de aquel país (Tedford et al., 2004); mientras que en México se han registrado en la porción temprana del Henfiliano tardío (Hh2) (6.95-5.59 Ma) de Zacatecas (Carranza-Castañeda, Aranda-Gómez, Wang, \& Iriondo, 2013). En EE. UU. el équido Dinohippus mexicanus se conoce sólo en el Henfiliano más tardío (Hh4) (ca. 5.0 Ma), mientras que en México se registra desde el Hh2 y hasta el Hh4 (6.95-4.8 Ma) (Carranza-Castañeda et al., 2013).

El camélido Alforjas y el antilocápridoTexoceros han sido registrados en el Hh4 de Guanajuato (4.8 Ma) (Jiménez-Hidalgo, 2005); mientras que en las localidades estadounidenses ambos se extinguen durante el Hh3 (ca. 6.0 Ma) (Tedford et al., 2004); asimismo, los camélidos Blancocamelus meadei y Hemiauchenia gracilis están presentes en sedimentos del Blancano III de Guanajuato (4.7-3.3 Ma), mientras que en las faunas locales de EE. UU. aparecen en el Blancano V (2.5-1.9 Ma) (Jiménez-Hidalgo \& CarranzaCastañeda, 2010). 
Por su parte, los fechamientos radiométricos y los estudios paleomagnéticos realizados en algunas localidades mexicanas reflejan que el límite temporal entre las NALMA en México no es sincrónico con las edades geológicas establecidas en EE. UU. En Nevada se estableció un límite para el Henfiliano/Blancano en 4.98-5.0 Ma (Lindsay et al., 2002), mientras que en Chihuahua este límite se identificó cerca de los 4.97 Ma (Lindsay et al., 2006), muy cercano al anterior; dicho límite se estableció en Guanajuato entre los 4.7 y 4.8 Ma (Flynn et al., 2005).

La diferencia de intervalos geocronológicos de varios taxones presentes en México y EE. UU. sugiere que ya no deben ser utilizados como marcadores de esas edades de mamíferos tan a la ligera (Flynn et al., 2005); es mejor realizar dataciones absolutas con isótopos radioactivos como U-Pb o Ar-Ar. Incluso en EE. UU., el muestreo continuo de sus faunas locales ha extendido el alcance geocronológico de algunos taxa, como por ejemplo el úrsido Agriotherium, el cual era marcador del Henfiliano y que posteriormente fue descubierto en sedimentos del Blancano de Idaho (Samuels, Meachen-Samuels, \& Gensler, 2009).

Afortunadamente, en México existen mamíferos que continúan siendo buenos marcadores de edad, como los bisontes o los équidos (Bell et al., 2004; Carranza-Castañeda et al., 2013); empero, los recientes trabajos taxonómicos, en diferentes épocas geológicas y regiones geográficas del país (Carranza-Castañeda, 2016; McDonald \& CarranzaCastañeda, 2017; Rincón, Czaplewski, MontellanoBallesteros, \& Benammi 2016; Stinnesbeck et al., 2017; Wang y Carranza-Castañeda, 2008) están mostrando que el grado de disimilitud taxonómica y temporal entre los conjuntos de mamíferos estadounidenses y mexicanos es considerablemente mayor a lo que se pensó en un inicio.

\section{El bisonte americano en pastizales y zonas de vegetación abiertas}

Uno de los supuestos más extendidos en los estudios paleobiológicos era considerar que una especie herbívora se alimentaba del mismo recurso alimentario, independientemente de la zona geográfica en que habitara. En estudios recientes se ha visto que esto no siempre se cumple. El género Bison es de origen eurasiático y arribó a América del Norte a través del estrecho de Bering hace aproximadamente 200,000 años (Lange, 2002; McDonald, 1981). La única especie sobreviviente del Pleistoceno, el bisonte americano (Bison bison), es uno de los mamíferos terrestres más grandes de América del Norte, se alimenta primordialmente de pasto y es una especie clave de las praderas norteamericanas (Lange, 2002; Meagher, 1986) (figura 1 inciso A).

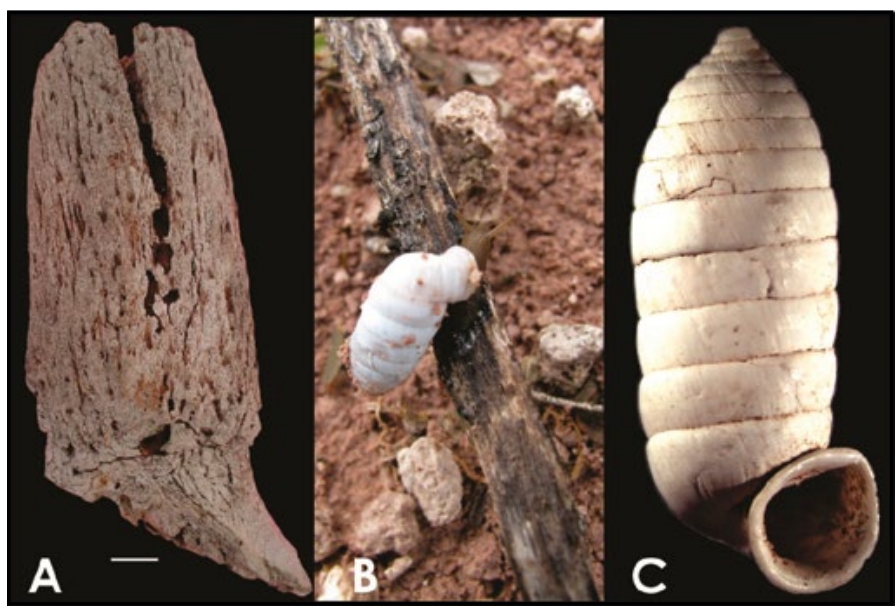

Figura 1. A) Núcleo de cuerno de Bison. B) Un caracol terrestre Holospira (Bostichocentrum) goldmani.

C) Concha de la misma especie de caracol.

Fotografías del equipo de investigación.

La condición especialista que distingue a los bisontes americanos actuales (pacedores y habitantes comunes de las praderas) generó la propuesta de que la presencia de fósiles de Bison sería indicativa de ambientes abiertos, cubiertos por pastos. Sin embargo, este planteamiento es inconsistente con información reciente sobre hábito alimentario mediante la caracterización de patrones de desgaste dental y composición isotópica de carbono $\left(\partial^{13} \mathrm{C}\right)$ en esmalte dental y/o hueso.

La evaluación microscópica y macroscópica del daño producido por el contacto diente/ diente (conocido como atrición) y diente/alimento (conocido como abrasión) en ejemplares de bisontes del Pleistoceno tardío de Alaska (Bison priscus), Nuevo México (Bison antiquus), así como de Texas y Florida (Bison sp.), y el centro y sur de México (B. antiquus) indica una dieta menos abrasiva que la reportada en los bisontes modernos, semejante a la de los herbívoros típicos con hábitos mixtos (Díaz-Sibaja, Jiménez-Hidalgo, Ponce-Saavedra, \& García Zepeda, 2018; Rivals, Solounias, \& Mihlbachler, 2007).

Por otra parte, la composición isotópica de carbono en muestras de Bison del Pleistoceno tardío 
issn 1665-4412, e-issn 2521-9758

Guerrero-Arenas, R., Jiménez-Hidalgo,

E., \& Bravo-Cuevas, V. M. de la costa de California, las Grandes Planicies, la Planicie Costera del Golfo y el centro de México presenta valores de ${ }^{13} \mathrm{C}$, que varían de $-2.0 \%$ a $-7.0 \%$, indicando una dieta variable consistente en plantas C3 y C4 (Bravo-Cuevas, Rivals, \& Priego-Vargas, 2017). Ello refleja un cambio de un comportamiento alimentario flexible a una dieta restringida de los bisontes extintos a los bisontes modernos. Es probable que esto se relacione con las modificaciones climáticas ocurridas en el Pleistoceno tardío y principios del Holoceno (Rivals et al., 2007). El amplio espectro alimentario observado en los bisontes pleistocénicos permitió a estos bóvidos ocupar diversos hábitats, incluso zonas de vegetación abierta; ambientes mixtos de zonas abiertas y arboladas, así como zonas arboladas (Bravo-Cuevas et al., 2017; Díaz-Sibaja et al., 2018).

\section{Un presente incompleto no puede ser (una) llave del pasado}

La forma en la que en el presente estudio se proponen las hipótesis de las condiciones de los ambientes antiguos es inductivo. Mientras mayor sea el conocimiento de las especies actuales conforme a su historia de vida las hipótesis tendrán mayor soporte y las aproximaciones estarán mejor sustentadas e incluso obliga a la revisión de paradigmas usados en la interpretación de condiciones del pasado. Por ejemplo, los gasterópodos terrestres han sido usados como indicadores (proxies) ecológicos debido a su aparente restricción a ciertas condiciones ambientales, como vegetación o suelo. Los caracoles urocóptidos son referidos como "obligados" de suelos con carbonato de calcio (Thomspon \& Mihalcik, 2005). Sin embargo, recientemente se reconocieron ejemplares de urocóptidos vivos (Holospira (Bostrichocentrum) goldmani) en suelos derivados de sedimentos volcánicos (Guerrero-Arenas, JiménezHidalgo, \& Sandoval-García, 2018) (figura 1 inciso B y figura 1 inciso C).

En los últimos años el conocimiento de la fisiología de los organismos y de su capacidad para adaptarse a diferentes ambientes ha aumentado considerablemente. Las interpretaciones de los hábitos dietarios en herbívoros (y, por ende, de la vegetación presente cuando el animal vivía) no consideran a la fisiología como un factor que determine el espectro de recursos alimentarios que pueda explotar. De manera general, la microbiota de los mamíferos está ligada a los cambios de hábito dietario a lo largo del tiempo (Ley et al., 2008).

Con base en diversas especies de rumiantes actuales (bovinos, ovejas, cabras, cérvidos y camellos) Henderson et al. (2015) demostraron que las diferencias en las comunidades microbianas del rumen pueden atribuirse a la dieta $y$, en menor medida, al huésped; la flexibilidad de la estructura de la comunidad microbiana del rumen puede conferirle a los rumiantes la habilidad de explotar una variedad de plantas como recursos alimenticios (Henderson et al., 2015), lo cual debe considerarse si se plantea que las especies recientes sean homólogas a las fósiles.

CONCLUSIONES

Es importante recalcar que las interpretaciones del pasado geológico deben realizarse con mayor rigor. El registro fósil es irremplazable en el conocimiento de la historia de la biota de nuestro planeta; las deficientes interpretaciones de estudios de biotas fósiles no invalida su importancia como fuente de información. Es imperativo que los paleontólogos no pasen por alto las investigaciones biológicas contemporáneas. Aunque la neontología y la paleontología se consideraron antaño como ciencias divorciadas (González Medrano, 1998), si ambas se integran la información generada será más robusta e integral (Hlusko, Schmitt, Monson, Brasil, \& Mahaney 2016; Feduccia, Lingham-Soliar, \& Hinhliffe, 2005; SánchezVillagra, 2010; Sarzetti, Dinghi, Genise, Bedatou, \& Verde, 2013).

El registro fósil mexicano destaca por su singularidad. Existen cada vez más evidencias de que los conjuntos de vegetación del pasado carecen de análogos modernos en varios periodos, incluso en aquellos relativamente cercanos, como el Pleistoceno tardío o el Holoceno (CaballeroRodríguez et al., 2017; Correa-Metrio et al., 2012; Cruz, Arroyo-Cabrales, \& Reynoso, 2016). Es deseable que las generaciones futuras de paleontólogos y estudiosos de las ciencias de la Tierra opten por una postura crítica con respecto a las hipótesis propuestas, en referencia a las condiciones ambientales del pasado en territorio mexicano y a los procedimientos utilizados para generarlas. 


\section{REFERENCIAS}

- Baker, V. R. (2014). Uniformitarianism, earth system science, and geology. Anthropocene, 5, 76-79. doi: 10.1016/j. ancene.2014.09.001

- Balashov, Y. (1994). Uniformitarianism in Cosmology: Background and philosophical implications of the steadystate theory. Studies in History and Philosophy of Science Part A, 25(6), 933-958. doi: 10.1016/0039-3681(94)90069-8

- Bell, C. J., Lundelius Jr., E. L., Barnosky, A. D., Graham, R.W., Lindsay, E. H., Ruez, D. R., ... Zakrzewski, R. J. (2004). The Blancan, Irvingtonian, and Rancholabrean mammal ages. En M. O. Woodburne (Ed.), Late Cretaceous and Cenozoic mammals of North America (pp. 232-314). New York: Columbia University Press.

- Bravo-Cuevas, V. M., Rivals, F., \& Priego-Vargas, J. (2017). Paleoecology $\left(\partial^{13} \mathrm{C}\right.$ and $\partial^{18} \mathrm{O}$ stable isotopes analysis) of a mammalian assemblage from the late Pleistocene of Hidalgo, central Mexico and implications for a better understanding of environmental conditions in temperate North America $\left(18^{\circ}-36^{\circ} \mathrm{N}\right.$ Lat.). Palaeogeography, Palaeoclimatology, Palaeoecology, 485, 632-643. doi: 10.1016/j.palaeo.2017.07.018

- Caballero-Rodríguez, D., Lozano-García, S., \& Correa-Metrio, A. (2017). Vegetation assemblages of central Mexico through the late Quaternary: Modern analogs and composition turnover. Journal of Vegetation Science, 28(3), 504-514. doi: $10.1111 /$ jvs. 12515

- Carranza-Castañeda, O. (2016). Roedores caviomorfos (Rodentia: Hydrochoeridae) del Blancano temprano-tardíoIrvingtoniano de los estados de Guanajuato, Jalisco y Sonora, México: Relación con Phugatherium dichroplax. Revista Mexicana de Ciencias Geológicas, 33(3), 297-315. doi: 10.22201/cgeo.20072902e.2016.3.445

- Carranza-Castañeda, O., Aranda-Gómez, J. J., Wang, X., \& Iriondo, A. (2013). The early-late Hemphillian (Hh2) faunal assemblage from Juchipila Basin, state of Zacatecas, Mexico, and its biochronologic correlation with other hemphillian faunas in central Mexico. Contributions in Science, 521, 13-49.

- Ceballos, G., Arroyo-Cabrales, J., \& Ponce, E. (2010). Effects of Pleistocene environmental changes on the distribution and community structure of the mammalian fauna of Mexico. Quaternary Research, 73(3), 464-473. doi: 10.1016/j. yqres.2010.02.006

- Cevallos-Ferriz, S. R. S., \& González-Torres, E. A. (2005). Geological setting and phytodiversity in Mexico. En F. J. Vega et al. (Eds.), Studies on Mexican Paleontology (pp. 1-18). Dordrecht: Springer.
- Cevallos-Ferriz, S. R. S., González-Torres, E. A., \& CalvilloCanadell, L. (2012). Perspectiva paleobotánica y geológica de la biodiversidad en México. Acta Botánica Mexicana, $100,317-350$.

- Correa-Metrio, A., Bush, M. B., Cabrera, K. R., Sully, S., Brenner, M., Hodell, D. A., ... Guilderson, T. (2012). Rapid climate change and no-analog vegetation in lowland Central America during the last 86,000 years. Quaternary Science Review, 38, 63-75. doi: 10.1016/j.quascirev.2012.01.025

- Cronin, T. M. (2010). Paleoclimates. Understanding climate change past and present. US: Columbia University Press.

- Cruz, J. A., Arroyo-Cabrales, J., \& Reynoso, V. H. (2016). Reconstructing the paleoenvironment of Loltún Cave, Yucatán, México, with Pleistocene amphibians and reptiles and their paleobiogeographic implications. Revista Mexicana de Ciencias Geológicas, 33(3), 342-354. doi: 10.22201/ cgeo.20072902e.2016.3.441

- De Francesco, C. G. (2013). PALEOLIMNOLOGY. Freshwater mollusks. En S. A. Elias, \& C. J. Mock (Eds.), Encyclopedia of Quaternary Science (pp. 281-291). United Kingdom: Elsevier.

- Díaz-Sibaja, R., Jiménez-Hidalgo, E., Ponce-Saavedra, J., \& García Zepeda, M. A. (2018). A combined mesowear analysis of Mexican Bison antiquus shows a generalist diet with geographical variation. Journal of Paleontology, 92 (6), 1130 1139. doi: 10.1017/jpa.2018.19

- Feduccia, A., Lingham-Soliar, T. \& Hinhliffe, J. R. (2005). Do feathered dinosaurs exist? Testing the hypothesis on neontological and paleontological evidence. Journal of Morphology, 266(2), 125-166. doi: 10.1002/jmor.10382

- Ferrari, L. (2017). Geología y biodiversidad: Una evolución paralela. En S. R. S. Cevallos-Ferriz, \& A. R. Huerta-Vergara (Eds.), Paleobiología. Interpretando procesos de la vida pasada (pp. 237-256). México: UNAM.

- Flynn, J. J., Kowallis, B. J., Nuñez, C., Carranza-Castañeda, O., Miller, W. E., Swisher, C. C., \& Lindsay, E. (2005). Geochronology of Hemphillian-Blancan aged strata, Guanajuato, Mexico, and implications for timing of the Great American Biotic Interchange. The Journal of Geology, 113(3), 287-307. doi: $10.1086 / 428806$

- Flores-Villela, O., \& Martínez-Salazar, E. A. (2009). Historical explanation of the origin of the herpetofauna of Mexico. Revista Mexicana de Biodiversidad, 80(3), 817-833.

- García-Cruz, C. M. (1999). El principio de Uniformidad (II). Un obstáculo epistemológico entre el pasado y el presente. Enseñanza de las Ciencias de la Tierra, 7.1, 16-20.

- González Medrano, F. (1998). La paleontología y la distribución de los organismos. Ciencias, 52, 72-82. 
- Gould, S. J. (1965). Is uniformitarianism necessary? American Journal of Science, 263(3), 223-228. doi: 10.2475/ajs.263.3.223 (1987). Time's arrow, time's cycle. Myth and metaphor in the discovery of geological time. Cambridge: Harvard University Press.

- Guerrero-Arenas, R., Jiménez-Hidalgo, E., \& Sandoval-García, C. (2018). New occurrences of Holospira (Bostrichocentrum) goldmani Bartsch, 1906 (Gastropoda: Urocoptidae) in northwestern Oaxaca, southern Mexico. Check List, 14(1), 107-112. doi: 10.15560/14.1.107

- Harrison, S. (2012). Philosophical and methodological perspectives on the science of environmental change. En J. A Matthews et al. (Eds.), The SAGE Handbook of Environmental Change (pp. 37-52). United Kingdom: SAGE. doi: $10.4135 / 9781446253045 . n 2$

- Henderson, G., Cox, F., Ganesh, S., Jonker, A., Young, W., Global Rumen Census Collaborators, \& Janssen, P. H. (2015). Rumen microbial community composition varies with diet and host, but a core microbiome is found across a wide geographical range. Scientific Reports, 5, 14567. doi: 10.1038/ srepl 4567

- Hlusko, L. J., Schmitt, C. A., Monson, T. A., Brasil, M. F., \& Mahaney, M. C. (2016). The integration of quantitative genetics, paleontology, and neontology reveals genetic underpinnings of primate dental evolution. Proceedings of National Academy of Sciences of the United States of America, 113(33), 9262-9267. doi: 10.1073/pnas.1605901113

- Hulbert, R. C. (2015). En Encyclopedia of Florida Vertebrate Paleontology Land Mammal Ages. Gainesville, FL: Florida Museum of Natural History/University of Florida. Recuperado de https://www.floridamuseum.ufl.edu/florida-vertebratefossils/land-mammal-ages/

- Jiménez-Hidalgo, E. (2005). Bioestratigrafía de los artiodáctilos (Tylopoda y Ruminantia) de las faunas del Terciario tardío de la Faja Volcánica Transmexicana (Tesis doctoral). Universidad Nacional Autónoma de México, México. Recuperada de https://repositorio.unam.mx/contenidos/63006

- Jiménez-Hidalgo, E., \& Carranza-Castañeda, O. (2010). Blancan camelids from San Miguel de Allende, Guanajuato, Central México. Journal of Paleontology, 84(1), 51-65. doi: $10.1666 / 08-154.1$

- Knight, J., \& Harrison, S. (2014). Limitations of uniformitarianism in the Anthropocene. Anthropocene, 5, 71-75. doi: 10.1016/j. ancene.2014.06.00

- Lange, I. M. (2002). Ice Age mammals of North America. Montana: Mountain Press Publishing Company.

- $\quad$ Ley, R. E., Hamady, M., Lozupone, C., Turnbaugh, P. J., Ramey,
R. R., Bircher, S., ... Gordon, J. I. (2008). Evolution of mammals and their gut microbes. Science, 320(5883), 1647-1651. doi: 10.1126/science. 1155725

- Lindsay, E. H. (2003). Chronostratigraphy, Biochronology, Datum Events, Land Mammal Ages, Stage of Evolution, and Appearance Event Ordination. Bulletin American Museum of Natural History, 279, 212-230.

- Lindsay, E. H., Mou, Y., Downs, W., Pederson, J., Kelly, T. S., Henry, C., \& Trexler, J. (2002). Recognition of the Hemphillian/Blancan boundary in Nevada. Journal of Vertebrate Paleontology, 22(2), 429-442. doi: 0.1671/0272-4634(2002)022[0429:ROTHBB] 2.0.CO;2

- $\quad$ Lluch-Cota, S., Saldívar-Lucio, R., \& Aranceta-Garza, F. (2017). El Antropoceno: ¿Una nueva era del planeta? Ciencias, 125, 4-9.

- Louys, J., Wilkinson, D. M., \& Bishop, L. C. (2012). Ecology needs a paleontological perspective. En J. Louys (Ed.), Paleontology in Ecology and Conservation (pp. 23-38). Berlin-Heidelberg: Springer. doi: 10.007/978-3-642-25038-5_3

- Lowe, J. J., \& Walker, M. J. C. (2015). Reconstructing Quaternary environments. India: Routledge-Taylor and Francis Group.

- McDonald, J. N. (1981). North American Bison: Their Classification and Evolution. Berkeley: University of California Press.

- McDonald, H. G., \& Carranza-Castañeda, O. (2017). Increased xenarthran diversity of the Great American Biotic Interchange: A new genus and species of ground sloth (Mammalia, Xenarthra, Megalonychidae) from the Hemphillian (late Miocene) of Jalisco, Mexico. Journal of Paleontology, 91 (5), 1069-1082. doi: 10.1017/jpa.2017.45

- Meagher, M. (1986). Bison bison. Mammalian species, 266, 1-8.

- Ramírez-Barahona, S., \& Eguiarte, L. E. (2013). The role of glacial cycles in promoting genetic diversity in the Neotropics: The case of cloud forests during the Last Glacial Maximum. Ecology and Evolution, 3(3), 725-738. doi: 10.1002/ece3.483

- Rincón, A. D., Czaplewski, N. J., Montellano-Ballesteros, M., \& Benammi, M. (2016). New species of Postcopemys (Cricetidae: Rodentia) from the early Pliocene of Lago de Chapala, Jalisco, Mexico. The Southwestern Naturalist, 61 (2), 108-118. doi: 10.1894/15-00082R2.1

- Rivals, F., Solounias, N., \& Mihlbachler, M. C. (2007). Evidence for geographic variation in the diets of late Pleistocene and early Holocene Bison in North America, and differences from the diets of recent Bison. Quaternary Research, 68(3), 338-346. doi: 10.1016/j.yqres.2007.07.012

- Romano, M. (2015). Reviewing the term uniformitarianism in 
modern Earth sciences. Earth-Science Reviews, 148, 65-76. doi: 10.1016/j.earscirev.2015.05.010

- Samuels, J. X., Meachen-Samuels, J. A., \& Gensler, P. A. (2009). The first mid-Blancan occurrence of Agriotherium (Ursidae) in North America: A record from Hagerman Fossil Beds National Monument, Idaho. Journal of Paleontology, 83(4), 597-603. doi: 10.1666/08-112R.1

- Sánchez-Villagra, M. R. (2010). Suture closure as a paradigm to study late growth in recent and fossil mammals: A case study with giant deer and dwarf deer skulls. Journal of Vertebrate Paleontology, 30, 1895-1898. doi: $10.1080 / 02724634.2010 .521218$

- $\quad$ Sarzetti, L. C., Dinghi, P. A., Genise, J. F., Bedatou, E., \& Verde, M. (2013). Curved fossil bee cells as tools for reconstructing the evolutionary history and palaeogeographical distribution of Diphaglossinae (Apoidea, Colletidae). Palaeontology, 57(2), 447-455. doi: 10.1111/pala.12074

- $\quad$ Shea, J.H.(1982).Twelve fallacies of uniformitarianism.Geology, 10(9), 455-460. doi: 10.1130/0091-7613(1982) 10<455:TFOU>2.0. $\mathrm{CO} ; 2$

- Stinnesbeck, S. R., Frey, E., Avíles Olguín, J., Stinnesbeck, W., Zell, P., Mallison, H., ... \& Rojas Sandoval, C. (2017). Xibalbaonyx oviceps, a new megalonychid ground sloth (Folivora, Xenarthra) from the Late Pleistocene of the Yucatán Peninsula, Mexico, and its paleobiogeographic significance. PalZ, 91, 245-271. doi: 10.1007/s12542-017-0349-5

- Tedford, R. H., Albright, L. B., Barnosky, A. D., FerrusquíaVillafranca, I., Hunt, R. M., Storer, J. E., ... Whistler, D. P. (2004). Mammalian biochronology of the Arikareean through Hemphillian intervals (late Oligocene through early Pliocene epochs). En M. O. Woodburne (Ed.), Late Cretaceous and Cenozoic mammals in North America: Biostratigraphy and geochronology (pp. 169-231). New York: Columbia University Press.

- Thompson, F. G., \& Mihalcik, E. L. (2005). Urocoptid landsnails of the genus Holospira from southern Mexico. Bulletin of the Florida Museum of Natural History (pp. 63-124). Gainesville, FL: University of Florida.

- Wang, X., \& Carranza-Castañeda, O. (2008). Earliest hognosed skunk, Conepatus (Mephitidae, Carnivora), from the early Pliocene of Guanajuato, Mexico and origin of South American skunks. Zoological Journal of the Linnean Society, 154 (2), 386-407. doi: 10.1111/j.1096-3642.2008.00411.x

- Woodburne, M. O. (2004). Late Cretaceous and Cenozoic mammals of North America: Biostratigraphy and Geochronology. US: Columbia University Press. 